\title{
The Effect of Tick Size on Testing for Nonlinearity in Financial Markets Data
}

\author{
Heather Mitchell ${ }^{1}$, Michael McKenzie ${ }^{2,3}$ \\ ${ }^{1}$ School of Economics, Finance and Marketing, Royal Melbourne Institute of Technology, Melbourne, Australia \\ ${ }^{2}$ Faculty of Economics and Business, University of Sydney, Sydney, Australia \\ ${ }^{3}$ Centre for Financial Analysis and Policy, Cambridge University, Cambridge, UK \\ E-mail: heather.mitchell@rmit.edu.au,michael.mckenzie@sydney.edu.au \\ Received May 6, 2011; revised May 16, 2011; accepted May 19, 2011
}

\begin{abstract}
The discrete nature of financial markets time-series data may prejudice the BDS and Close Returns test for nonlinearity. Our estimation results suggest that a tick/volatility ratio threshold exists, beyond which the test results are biased. Further, tick/volatility ratios that exceed these thresholds are frequently observed in financial markets data, which suggests that the results of the BDS and CR test must be interpreted with caution.
\end{abstract}

Keywords: Compass Rose, Tick/Volatility Ratio, BDS Test, Close Returns Test

\section{Introduction}

A body of literature has evolved which considers the implications of discreteness for: 1) trading behaviour [1]; 2) technical trading strategies [2,3]; 3) model estimation [4,5]; and most importantly in the current context, 4) tests of the statistical properties of financial market data. For example, Gottleib and Kalay [6] find that discreteness biases the second and higher order moment estimates of returns data upwards. Koppl and Tuluca and Fang $[7,8]$ consider the impact of the compass rose on random walk testing. Crack and Ledoit [9] discuss how the presence of the compass rose pattern may distort the null distribution for the Brock, Dechert and Scheinkman (BDS) test for nonlinearity. Kramer and Runde [10] empirically test this proposition and find that the null distribution of the BDS test for chaos is distorted in the presence of discreteness.

The purpose of this paper is to provide further evidence of the impact of discreteness on tests of the properties of financial markets data. Specifically, the BDS test of Brock, Dechert and Scheinkman [11] and the Close Returns (CR hereafter) tests of nonlinearity are considered. The results suggest that both the CR and BDS test are sensitive to data discreteness, although only in sample sizes of less than 500 observations for the latter.

\section{The BDS and Close Returns Test}

One of the most commonly applied tests for nonlinearity is the BDS test of Brock, Dechert and Scheinkman [11] details of which may be found in Dechert [12]. In essence, the test simply focuses on pairs of points in the dataset. If the series is iid, then the probability of the distance between these points being less than or equal to some arbitrarily chosen distance, $\varepsilon$ will be a constant. More formally, the BDS test is a statistical test of the null hypothesis of IID and is based on the Grassberger and Procaccia [13] correlation integral for a given embedding dimension. If the test value is significantly different from a standard normal distribution, it can be concluded that the given signal is deterministic. As such, the BDS procedure may be considered as a test for linear and nonlinear departures from IID rather than a specific test for chaos. It is in this latter context however, that the test has most commonly been applied usually in conjunction with the estimation of entropy, Lyapunov exponents or correlation dimensions. The BDS test belongs to the metric invariant class of tests for chaotic behaviour and an extensive literature has emerged which uses the BDS metric to test for nonlinear behaviour in a wide range of financial data including: 1) stock market returns [14,15], 2) exchange rates $[16,17], 3)$ futures data [18], 4) commodity prices $[19,20]$ and 5) macroeconomic data [21]. In general, the BDS test results furnished by this literature provide substantial empirical evidence of nonlinear structure in a wide range of financial asset prices.

An alternative nonlinear testing procedure is the Close Returns (CR hereafter) test details of which may be found in Gilmore [22]. The close returns test is a topo- 
logical based testing procedure which was specifically designed to detect low dimensional chaotic behaviour. It is a two-part test consisting of a qualitative component which is a graphical test for the presence of chaotic behaviour. The second quantitative element is a test of the null hypothesis that the data is IID against both linear and nonlinear alternatives. The topological approach attempts to determine how the unstable periodic orbits of the strange attractor are intertwined. The processes of stretching and compression are responsible for organising the strange attractor in a unique way and if one can determine how the unstable periodic orbits are organised, we can identify the stretching and compressing mechanisms responsible for the creation of the strange attractor. This information is robust against noise and is independently verifiable. Once these mechanisms have been identified, a geometric model can be constructed which describes how to model the stretching and squeezing mechanisms responsible for generating the original time series. That is to say, topological tests may not only detect the presence of chaos (the only information provided by the metric class of tests), but can also provide information about the underlying system responsible for the chaotic behaviour. As the topological method preserves the time ordering of the data, where evidence of chaos is found, the researcher may proceed to characterise the underlying process in a quantitative way. Thus, one is able to reconstruct the stretching and compressing mechanisms responsible for generating the strange attractor. While not enabling the researcher to identify the underlying equation system, it does allow the rejection of models which produce behaviour that is incompatible with the characteristics of the strange attractor identified by this technique. Applications of the CR test to financial markets data may be found in Gilmore and McKenzie [17,22-26].

\section{Random Data and the BDS and Close Returns Test}

To assess the impact of discreteness of the BDS and CR test, we must firstly benchmark the performance of these tests against simulated data. As such, a 1,000 observation series of random numbers is created using the Knuth [27] psuedo-random number generator, which is assumed to be normally distributed with a zero mean and constant variance equal to one. ${ }^{1}$ The BDS test and CR test are then applied to these data, which are recorded to 18 decimal

\footnotetext{
${ }^{1}$ To ensure that the results are driven by the tick effect and not the psuedo-randomness of the number generator, Knuth's [27] lagged Fibonacci generator, L'Ecuyer's [29] combined multiple recursive generator and Matsumoto and Nishimura's [30] Mersenne Twister are all considered. All three generators produced qualitatively consistent results and so, to conserve space, the discussion shall focus solely on the Knuth results.
}

places.

When estimating the BDS test, the neighbourhood size and the embedding dimension must be specified. As a general guide, Brock, Hsieh and LeBaron [28] suggest an embedding dimension of between 2 and 5 and a range for the threshold term of 0.5 to 2 . In an effort to make our results as generalisable as possible, we extent the range of values suggested by Brock et al. [28] and estimate the BDS test assuming an embedding dimension of 2 - 6 and a threshold term which ranges from 0.01 to 5 . $^{2}$ Thus, the BDS test is applied to each 1,000 observation simulated data series assuming each combination of embedding dimension and threshold term. This process is repeated 1,000 times and Panel A of Table 1 presents a summary of the proportion of rejections of the null. The nominal size of the test is assumed to be 0.05 in all cases and the figures in bold indicate the instances in which the proportion of rejections of the null is not significantly different from the nominal size of the level of significance.

The performance of the CR test when applied to a random number series may also be considered. The CR test requires the neighbourhood and histogram size to be specified. Both of these values are subjective and a histogram length of between 400 and 600 observations is assumed. Further, the neighbourhood size is assumed to vary over the same range as set in the BDS test results. The estimation results are presented in Panel B of Table $\mathbf{1}$ and the figures in bold indicate the instances in which the proportion of rejections of the null is not significantly different from the nominal size of the level of significance.

In general, the results suggest that both the BDS and CR test are sensitive to the specification of the neighurhood size. The BDS test performs best for a threshold value of 1.00 or 1.50 whereas the CR test performs best for a neighbourhood size of 0.01 to 0.05 . Neither test performs well for values outside of this range as the BDS test fails to reject the null sufficiently often, whereas the CR test almost never fails to reject the null. The choice of embedded dimension for the BDS test, or histogram size for the CR test, do not appear to impact on the general tenor of the results.

\section{Discrete Data and the BDS and Close Returns Test}

The extent to which discreteness imposes itself on the movements in prices is a function of the volatility of the data. As such, the performance of the BDS and CR test will be considered for simulated data that exhibits dif-

${ }^{2}$ A neighbourhood size of 7.5 and 10 was also specified, however the BDS typically failed to furnish a result and so presenting information as to the proportion of rejections of the null is not informative. 
Table 1. Performance of BDS and CR test.

\begin{tabular}{ccccccccccccc}
\hline Neighbour-hood Size & \multicolumn{3}{c}{ Panel A: Knuth Generator Embedding Dimension } & \multicolumn{3}{c}{ Panel B: Knuth Generator Histogram Size } \\
\hline & 2 & 3 & 4 & 5 & 6 & 400 & 450 & 500 & 550 & 600 \\
\hline 0.01 & 0.862 & 1.000 & 1.000 & 1.000 & 1.000 & $\mathbf{0 . 0 4 1}$ & $\mathbf{0 . 0 4 4}$ & 0.033 & $\mathbf{0 . 0 3 6}$ & $\mathbf{0 . 0 4 4}$ \\
0.05 & 0.642 & 0.858 & 1.000 & 1.000 & 1.000 & $\mathbf{0 . 0 3 9}$ & $\mathbf{0 . 0 4 2}$ & $\mathbf{0 . 0 3 7}$ & $\mathbf{0 . 0 4 1}$ & 0.025 \\
0.10 & 0.385 & 0.670 & 0.851 & 1.000 & 1.000 & 0.026 & 0.031 & 0.028 & 0.024 & 0.021 \\
0.25 & 0.140 & 0.226 & 0.395 & 0.622 & 0.798 & 0.010 & 0.014 & 0.009 & 0.012 & 0.017 \\
0.50 & 0.076 & 0.094 & 0.093 & 0.131 & 0.191 & 0.005 & 0.006 & 0.002 & 0.002 & 0.006 \\
0.70 & $\mathbf{0 . 0 6 2}$ & 0.065 & $\mathbf{0 . 0 6 0}$ & $\mathbf{0 . 0 6 2}$ & 0.079 & 0.001 & 0.003 & 0.002 & 0.005 & 0.003 \\
1.00 & $\mathbf{0 . 0 5 2}$ & $\mathbf{0 . 0 6 0}$ & $\mathbf{0 . 0 6 3}$ & $\mathbf{0 . 0 6 4}$ & $\mathbf{0 . 0 6 3}$ & 0.000 & 0.000 & 0.000 & 0.001 & 0.001 \\
1.50 & $\mathbf{0 . 0 5 1}$ & $\mathbf{0 . 0 5 5}$ & $\mathbf{0 . 0 5 2}$ & $\mathbf{0 . 0 5 5}$ & $\mathbf{0 . 0 5 5}$ & 0.000 & 0.000 & 0.000 & 0.002 & 0.007 \\
2.00 & $\mathbf{0 . 0 5 6}$ & 0.069 & $\mathbf{0 . 0 6 2}$ & 0.065 & 0.073 & 0.000 & 0.000 & 0.000 & 0.001 & 0.007 \\
2.50 & 0.072 & 0.075 & 0.069 & 0.066 & 0.061 & 0.000 & 0.000 & 0.002 & 0.002 & 0.004 \\
3.00 & 0.092 & 0.087 & 0.106 & 0.095 & 0.103 & 0.000 & 0.000 & 0.000 & 0.005 & 0.005 \\
4.00 & 0.131 & 0.182 & 0.210 & 0.234 & 0.258 & 0.004 & 0.001 & 0.002 & 0.005 & 0.007 \\
5.00 & 0.177 & 0.250 & 0.308 & 0.361 & 0.411 & 0.018 & 0.020 & 0.016 & 0.015 & 0.017 \\
\hline
\end{tabular}

Figures in bold indicate the proportion of rejections of the null is not significantly different from the nominal size ( $\alpha=0.05)$ testing at a level of significance of 0.05 .

ferent tick to volatility ratios. ${ }^{3}$ The Knuth generator is used to generate a simulated series of 1,000 random observations drawn from a normal distribution with a zero mean and constant unit variance. The data was then 'discretised' to produce a given tick/volatility ratio by allocating each observation to a bin (as is done for a histogram), where the size of the bins is determined by the tick size. To make our results as generalisable as possible, the lower bound for the tick/volatility ratio is set at 0.01 which is well below the smallest tick/volatility ratios typically observed in financial markets (Gleason, Lee and Mathur, 2000, report tick/volatility ratios as low as 0.167 for exchange rate data). The upper bound is set at 5.0 which is the maximum distance we expect to find between any two data points in a standard normal distribution.

The filtered data is tested for the presence of nonlinearities using the BDS and CR test. As a control, the raw data is also included in the analysis. The BDS test is applied assuming a dimension range of between 2 and 6 and the test neighbourhood size is set at between 0.70 and 1.5. This corresponds to the range of values for which the test worked well as discussed in Section 3. The

\footnotetext{
${ }^{3}$ Lee, Gleason and Mathur [31] find that the tick/volatility ratio is an important determinant of the visibility of the compass rose pattern as it determines the potential number of rays on which the data may fall and so, the obscurity of the pattern. Time is also important as a sufficiently long sample period is required to allow the phase portrait to fully evolve [32].
}

results are presented in Table 2 and the raw data provides a benchmark against which the results may be assessed. Across the four neighbour sizes and five dimensions considered, the proportion of rejections of the null for BDS test ranged from 0.049 to 0.081 . Modifying the data to exhibit a tick/volatility ratio of 0.01 does not serve to initiate any substantial changes in the performance of the BDS test. The null hypothesis is rejected in almost the same proportion as where the raw data is specified. As the tick/volatility ratio is increased, the proportion of times the null is rejected does not deviate substantially from the benchmark. Thus, the BDS test appears to work well for 1,000 observations data sets which exhibits reasonably high tick/volatility ratios.

The literature suggests the use of relatively small data sets may lead to spurious BDS test results [33-35]. Therefore, it is interesting to consider random number datasets of different lengths to test the robustness of the above results. We find that where a 500 observation series is considered, the results are broadly consistent with those previously reported. Where a 50, 100 and 250 observation random number data set is tested however, the BDS test applied to the raw data series consistently rejected the null a greater proportion of the time than is expected given the power of the test.

The impact of the tick/volatility ratio on the performance of the CR test is presented in Table 3 for 1,000 observation random number series. Guided by the results of 
Table 2. Effect of tick size on BDS test - Series length $=\mathbf{1 0 0 0}$.

\begin{tabular}{|c|c|c|c|c|c|c|c|c|c|c|c|c|c|c|}
\hline \multicolumn{15}{|c|}{ Tick Volatility Ratio } \\
\hline & None & 0.01 & 0.03 & 0.05 & 0.10 & 0.25 & 0.50 & 0.70 & 1.0 & 1.5 & 2.0 & 2.5 & 3.0 & 5.0 \\
\hline 2 & 0.060 & 0.061 & 0.062 & 0.058 & 0.061 & 0.064 & 0.056 & 0.061 & 0.070 & 0.050 & 0.056 & 0.051 & 0.051 & 0.086 \\
\hline 3 & 0.064 & 0.065 & 0.065 & 0.057 & 0.056 & 0.064 & 0.059 & 0.052 & 0.064 & 0.052 & 0.056 & 0.054 & 0.052 & 0.065 \\
\hline 4 & 0.063 & 0.063 & 0.068 & 0.065 & 0.069 & 0.072 & 0.066 & 0.054 & 0.082 & 0.055 & 0.047 & 0.058 & 0.052 & 0.051 \\
\hline 5 & 0.064 & 0.067 & 0.065 & 0.068 & 0.073 & 0.082 & 0.072 & 0.060 & 0.126 & 0.051 & 0.047 & 0.053 & 0.049 & 0.044 \\
\hline 6 & 0.081 & 0.083 & 0.081 & 0.084 & 0.084 & 0.105 & 0.066 & 0.060 & 0.195 & 0.058 & 0.046 & 0.049 & 0.050 & 0.047 \\
\hline Dim & \multicolumn{14}{|c|}{ Test Neighbourhood Size $=1.0$} \\
\hline 3 & 0.061 & 0.060 & 0.060 & 0.051 & 0.046 & 0.053 & 0.056 & 0.052 & 0.052 & 0.052 & 0.056 & 0.054 & 0.052 & 0.065 \\
\hline 4 & 0.052 & 0.051 & 0.050 & 0.053 & 0.052 & 0.054 & 0.054 & 0.054 & 0.049 & 0.055 & 0.047 & 0.058 & 0.052 & 0.051 \\
\hline 5 & 0.055 & 0.054 & 0.056 & 0.054 & 0.059 & 0.059 & 0.053 & 0.060 & 0.056 & 0.051 & 0.047 & 0.053 & 0.049 & 0.044 \\
\hline 6 & 0.062 & 0.059 & 0.059 & 0.055 & 0.057 & 0.051 & 0.050 & 0.060 & 0.056 & 0.058 & 0.046 & 0.049 & 0.050 & 0.047 \\
\hline Dim & \multicolumn{14}{|c|}{ Test Neighbourhood Size $=1.25$} \\
\hline 2 & 0.056 & 0.058 & 0.060 & 0.049 & 0.060 & 0.052 & 0.053 & 0.061 & 0.057 & 0.050 & 0.056 & 0.051 & 0.051 & 0.086 \\
\hline 3 & 0.057 & 0.054 & 0.056 & 0.048 & 0.053 & 0.056 & 0.056 & 0.052 & 0.052 & 0.052 & 0.056 & 0.054 & 0.052 & 0.065 \\
\hline 4 & 0.054 & 0.052 & 0.053 & 0.049 & 0.055 & 0.060 & 0.054 & 0.054 & 0.049 & 0.055 & 0.047 & 0.058 & 0.052 & 0.051 \\
\hline 5 & 0.061 & 0.058 & 0.057 & 0.053 & 0.057 & 0.059 & 0.053 & 0.060 & 0.056 & 0.051 & 0.047 & 0.053 & 0.049 & 0.044 \\
\hline Dim & \multicolumn{14}{|c|}{ Test Neighbourhood Size = 1.5} \\
\hline 2 & 0.049 & 0.046 & 0.045 & 0.046 & 0.049 & 0.048 & 0.049 & 0.051 & 0.057 & 0.056 & 0.056 & 0.051 & 0.051 & 0.086 \\
\hline 3 & 0.057 & 0.056 & 0.056 & 0.050 & 0.046 & 0.054 & 0.052 & 0.055 & 0.052 & 0.053 & 0.056 & 0.054 & 0.052 & 0.065 \\
\hline 4 & 0.051 & 0.053 & 0.051 & 0.051 & 0.048 & 0.056 & 0.049 & 0.053 & 0.049 & 0.044 & 0.047 & 0.058 & 0.052 & 0.051 \\
\hline 5 & 0.058 & 0.057 & 0.051 & 0.053 & 0.046 & 0.056 & 0.052 & 0.052 & 0.056 & 0.060 & 0.047 & 0.053 & 0.049 & 0.044 \\
\hline 6 & 0.049 & 0.049 & 0.050 & 0.045 & 0.047 & 0.052 & 0.049 & 0.052 & 0.056 & 0.058 & 0.046 & 0.049 & 0.050 & 0.047 \\
\hline
\end{tabular}

Section 3, the results are estimated across neighbourhood sizes which range from 0.01 to 0.05 and histogram lengths which range from 400 to 600 . Unlike the BDS test results, the performance of the CR test when applied to the raw data is not significantly different to the assumed power of the test $(\alpha=5 \%)$ for neighbourhood sizes of 0.01 and 0.02 . Test neighbourhood sizes of 0.03 and 0.05 furnished results whereby the CR test frequently rejected the null significantly less than is expected. The imposition of low tick/volatility ratios does not cause the proportion of rejections of the null to alter substantially from the benchmark. Where the tick/vola- tility ratio exceeds 0.10 however, the proportion of rejections of the null declines in all cases. Thus, the CR test is less likely to detect nonlinearity for higher tick/volatility ratios. This test procedure is repeated for sample lengths of 50, 100, 250 and 500 observation data sets and a similar pattern is observed.

In general, these results suggest that both the BDS and CR test are sensitive to the discreteness of the data, albeit in slightly different forms. For sample sizes of 500 observations or more, the BDS test is not effected by rounding effects. For sample sizes of less than 500 observations however, a tick/volatility ratio equal to the test neighbourhood size marks a threshold beyond which the proportion of rejections of the null hypothesis for the BDS test declines. The results of Section 3 suggest that the BDS test works best where a neighbourhood size of 
Table 3. Effect of tick size on close returns test - Series length $=\mathbf{1 0 0 0}$.

\begin{tabular}{|c|c|c|c|c|c|c|c|c|c|c|c|c|c|c|}
\hline \multicolumn{15}{|c|}{ Tick Volatility Ratio } \\
\hline & None & 0.01 & 0.03 & 0.05 & 0.10 & 0.25 & 0.50 & 0.70 & 1.0 & 1.5 & 2.0 & 2.5 & 3.0 & 5.0 \\
\hline Cells & \multicolumn{14}{|c|}{ Test Neighbourhood Size $=0.01$} \\
\hline 400 & 0.046 & 0.046 & 0.054 & 0.045 & 0.041 & 0.032 & 0.014 & 0.007 & 0.004 & 0.000 & 0.000 & 0.000 & 0.001 & 0.004 \\
\hline 450 & 0.044 & 0.042 & 0.049 & 0.033 & 0.031 & 0.034 & 0.014 & 0.015 & 0.004 & 0.000 & 0.000 & 0.000 & 0.002 & 0.006 \\
\hline 500 & 0.053 & 0.047 & 0.051 & 0.042 & 0.032 & 0.032 & 0.017 & 0.011 & 0.005 & 0.002 & 0.001 & 0.004 & 0.002 & 0.008 \\
\hline 550 & 0.046 & 0.046 & 0.042 & 0.056 & 0.043 & 0.033 & 0.014 & 0.008 & 0.005 & 0.004 & 0.004 & 0.007 & 0.007 & 0.012 \\
\hline 600 & 0.047 & 0.054 & 0.055 & 0.046 & 0.045 & 0.026 & 0.015 & 0.008 & 0.003 & 0.007 & 0.007 & 0.008 & 0.013 & 0.019 \\
\hline Cells & \multicolumn{14}{|c|}{ Test Neighbourhood Size $=0.02$} \\
\hline 400 & 0.044 & 0.049 & 0.054 & 0.045 & 0.041 & 0.032 & 0.014 & 0.007 & 0.004 & 0.000 & 0.000 & 0.000 & 0.001 & 0.004 \\
\hline 450 & 0.041 & 0.046 & 0.049 & 0.033 & 0.031 & 0.034 & 0.014 & 0.015 & 0.004 & 0.000 & 0.000 & 0.000 & 0.002 & 0.006 \\
\hline 500 & 0.045 & 0.043 & 0.051 & 0.042 & 0.032 & 0.032 & 0.017 & 0.011 & 0.005 & 0.002 & 0.001 & 0.004 & 0.002 & 0.008 \\
\hline 550 & 0.042 & 0.048 & 0.042 & 0.056 & 0.043 & 0.033 & 0.014 & 0.008 & 0.005 & 0.004 & 0.004 & 0.007 & 0.007 & 0.012 \\
\hline 600 & 0.042 & 0.041 & 0.055 & 0.046 & 0.045 & 0.026 & 0.015 & 0.008 & 0.003 & 0.007 & 0.007 & 0.008 & 0.013 & 0.019 \\
\hline Cells & \multicolumn{14}{|c|}{ Test Neighbourhood Size $=0.03$} \\
\hline 400 & 0.031 & 0.033 & 0.034 & 0.045 & 0.041 & 0.032 & 0.014 & 0.007 & 0.004 & 0.000 & 0.000 & 0.000 & 0.001 & 0.004 \\
\hline 450 & 0.024 & 0.032 & 0.046 & 0.033 & 0.031 & 0.034 & 0.014 & 0.015 & 0.004 & 0.000 & 0.000 & 0.000 & 0.002 & 0.006 \\
\hline 500 & 0.034 & 0.038 & 0.034 & 0.042 & 0.032 & 0.032 & 0.017 & 0.011 & 0.005 & 0.002 & 0.001 & 0.004 & 0.002 & 0.008 \\
\hline 550 & 0.038 & 0.043 & 0.043 & 0.056 & 0.043 & 0.033 & 0.014 & 0.008 & 0.005 & 0.004 & 0.004 & 0.007 & 0.007 & 0.012 \\
\hline 600 & 0.043 & 0.043 & 0.040 & 0.046 & 0.045 & 0.026 & 0.015 & 0.008 & 0.003 & 0.007 & 0.007 & 0.008 & 0.013 & 0.019 \\
\hline Cells & \multicolumn{14}{|c|}{ Test Neighbourhood Size $=0.05$} \\
\hline 400 & 0.031 & 0.034 & 0.037 & 0.033 & 0.041 & 0.032 & 0.014 & 0.007 & 0.004 & 0.000 & 0.000 & 0.000 & 0.001 & 0.004 \\
\hline 450 & 0.039 & 0.028 & 0.035 & 0.031 & 0.031 & 0.034 & 0.014 & 0.015 & 0.004 & 0.000 & 0.000 & 0.000 & 0.002 & 0.006 \\
\hline 500 & 0.041 & 0.043 & 0.042 & 0.033 & 0.032 & 0.032 & 0.017 & 0.011 & 0.005 & 0.002 & 0.001 & 0.004 & 0.002 & 0.008 \\
\hline 550 & 0.032 & 0.039 & 0.031 & 0.028 & 0.043 & 0.033 & 0.014 & 0.008 & 0.005 & 0.004 & 0.004 & 0.007 & 0.007 & 0.012 \\
\hline 600 & 0.030 & 0.035 & 0.031 & 0.021 & 0.045 & 0.026 & 0.015 & 0.008 & 0.003 & 0.007 & 0.007 & 0.008 & 0.013 & 0.019 \\
\hline
\end{tabular}

between 0.70 and 1.5 is specified. Thus, the BDS test results may be biased when applied to data which exhibits a tick/volatility ratio of at least 0.70 . The CR test exhibits the same behaviour however, it is not sensitive to sample length. As the tick/volatility ratio increases beyond a threshold, the proportion of rejections of the null hypothesis declines. This threshold is sensitive to the sample length and for 1,000 observation data series is 0.10 and increases to 1.0 for 50 observation series.

\section{Conclusions}

The discrete nature of financial markets time-series data may prejudice tests which attempt to detect the presence of nonlinearities. In particular, where the tick/volatility ratio is high, price movements are frequently bounded by the minimum tick size which may obfuscate any subtle nonlinear patterns which would be otherwise present. This paper considers the performance of the BDS and CR tests when applied to discrete data. In general, the estimation results suggest that both the BDS and CR test are sensitive to data discreteness, albeit in slightly different forms. For sample sizes of 500 observations or more, the BDS test is not biased by rounding effects. For sample sizes of less than 500 observations however, a tick/volatility ratio equal to the test neighbourhood size marks a threshold beyond which the proportion of rejections of the null hypothesis for the BDS test declines. 
The CR test provides the same result however, it is not sensitive to sample length. As the tick/volatility ratio increases beyond a threshold, the proportion of rejections of the null hypothesis declines. This threshold is sensitive to the sample length and for 1,000 observation data series is 0.10 and increases to 1.0 for 50 observation series.

Tick/volatility ratios which exceed these thresholds are frequently observed in financial markets data, which suggests that the results of the BDS and CR test must be interpreted with caution. Estimates of tick/volatility ratios for exchange rate data range from 0.167 to 0.5 [36] and for stock market range from 0.5 to 4.5 where prices are quoted using decimals and 2.5 to 35 for those using $1 / 8^{\text {th }} \mathrm{s}$ [37]. Thus, financial markets data frequently exhibit tick/volatility ratios which exceed the threshold beyond which the BDS and PCR test are biased by the discrete nature of prices.

\section{References}

[1] V. R. Anshuman and A. Kalay, "Market Making with Discrete Prices,” The Review of Financial Studies, Vol. 11, No. 1, 1998, pp. 81-109. doi:10.1093/rfs/11.1.81

[2] C. Vorlow, "Stock Price Clustering and Discreteness: The 'Compass Rose' and Complex Dynamics,” Working Paper, University of Durham, Durham, 2004.

[3] A. Antoniou and C. E. Vorlow, "Price Clustering and Discreteness: Is There Chaos behind the Noise," Physica $A$, Vol. 348, 2005, pp. 389-403. doi:10.1016/j.physa.2004.09.006

[4] C. Lee, K. Gleason and I. Mathur, "The Tick/Volatility Ratio as a Determinant of the Compass Rose Pattern," European Journal of Finance, Taylor and Francis Journals, Vol. 11, No. 2, April 2005, pp. 93-109.

[5] H. Amilon, "GARCH Estimation and Discrete Stock Prices: An Application to Low-Priced Australian Stocks,” Economics Letters, Vol. 81, No. 2, 2003, pp. 215-222. doi:10.1016/S0165-1765(03)00172-1

[6] G. Gottleib and A. Kalay, "Implications of the Discreteness of Observed Stock Prices,” Journal of Finance, Vol. 40, No. 1, 1985, pp. 135-154. doi:10.2307/2328052

[7] R. Koppl and S. Tuluca, "Random Walk Hypothesis Testing and the Compass Rose,” Finance Letters, Vol. 2, No. 1, 2002, pp. 14-17.

[8] Y. Fang, "The Compass Rose and Random Walk Tests," Computational Statistics and Data Analysis, Vol. 39, No. 3, 2002, pp. 299-310. doi:10.1016/S0167-9473(01)00063-9

[9] T. F. Crack and O. Ledoit, "Robust Structure without Predictability: The Compass Rose Pattern of the Stock Market," Journal of Finance, Vol. 51, No. 2, 1996, pp. 751-762. doi:10.2307/2329379

[10] W. Kramer and R. Runde, "Chaos and the Compass Rose," Economics Letters, Vol. 54, No. 2, 1997, pp. 113-118. doi:10.1016/S0165-1765(97)00020-7
[11] W. Brock, W. Dechert and J. Scheinkman, "A Test for Independence Based on the Correlation Dimension," Working Paper, University of Wisconsin, Madison, 1987.

[12] W. D. Dechert, "Testing Time Series for Nonlinearities: The BDS Approach,” In: W. A. Kirman,et al., Eds. Nonlinear Dynamics and Economics: Proceedings of the 10th International Symposium in Economic Theory and Econometrics, Cambridge University Press, Cambridge, 1996

[13] P. Grassberger and I. Procacia, "Measuring the Strangeness of Strange Attractors,” Physica D: Nonlinear Phenomena, Vol. 9, No. 1-2, 1983, pp. 189-208.

[14] Y.-T. Chen and C.-M. Kuan, "Time Irreversibility and EGARCH Effects in US Stock Index Returns,” Journal of Applied Econometrics, Vol. 17, No. 5, 2002, pp. 565-578. doi:10.1002/jae.692

[15] R. Ostermark, J. Aaltonen, H. Saxen and K. Soderlund, "Nonlinear Modelling of the Finnihs Banking and Finance Branch Index,” European Journal of Finance, Vol. 10, No. 4, 2004, pp. 277-289. doi:10.1080/13518470210124641

[16] R. T. Baillie, A. A. Cecen and Y.-W. Han, "High Frequency Deutschemark-US Dollar Returns: FIGARCH Representation and Non Linearities," Multinational Finance Journal, Vol. 4, No. 3-4, 2000, pp. 247-268.

[17] C. G. Gilmore, “An Examination of Nonlinear Dependence in Exchange Rates Using Recent Methods from Chaos Theory," Global Finance Journal, Vol. 12, No. 1, 2001, pp. 139-151. doi:10.1016/S1044-0283(01)00018-7

[18] V. Chwee, “Chaos in Natural Gas Futures?” The Energy Journal, Vol. 19, No. 2, 1998, pp. 149-164. doi:10.5547/ISSN0195-6574-EJ-Vol19-No2-10

[19] M. Frank and T. Stengos, "Chaotic Dynamics in Economic Time Series,” Journal of Economic Surveys, Vol. 2, No. 2, 1988, pp. 103-133. doi:10.1111/j.1467-6419.1988.tb00039.x

[20] N. Kohzadi and M. S. Boyd, "Testing for Chaos and Nonlinear Dynamics in Cattle Prices," Canadian Journal of Agricultural Economics, Vol. 43, No. 3, 1995, pp. 475484. doi:10.1111/j.1744-7976.1995.tb00136.x

[21] P. M. Bodman, "Steepness and Deepness in the Australian Macroeconomy," Applied Economics, Vol. 33, No. 3, 2001, pp. 375-382. doi:10.1080/00036840122115

[22] C. G. Gilmore, “An Examination of Nonlinear Dependence in Exchange Rates Using Recent Methods from Chaos Theory," Global Finance Journal, Vol. 12, No. 1, 2001, pp. 139-151. doi:10.1016/S1044-0283(01)00018-7

[23] C. G. Gilmore, “A New Test for Chaos,” Journal of Economic Behaviour and Organisation, Vol. 22, No. 2, 1993, pp. 209-237. doi:10.1016/0167-2681(93)90064-V

[24] C. G. Gilmore, “A New Approach to Testing for Chaos, with Applications in Finance and Economics," International Journal of Bifurcation and Chaos, Vol. 3, No. 3, 1993, pp. 583-587. doi:10.1142/S0218127493000477

[25] C. G. Gilmore, "Detecting Linear and Nonlinear Dependence in Stock Returns: New Methods Derived from Chaos Theory," Journal of Business and Finance Ac- 
counting, Vol. 23, No. 9-10, 1996, pp. 1357-1377. doi:10.1111/1468-5957.00084

[26] M. D. McKenzie, "Chaotic Behaviour in National Stock Market Indices: New Evidence from the Close Returns Test," Global Finance Journal, Vol. 12, No. 1, 2001, pp. 35-53. doi:10.1016/S1044-0283(01)00024-2

[27] D. E. Knuth, “The Art of Computer Programming,” 3rd Edition, Addison-Wesley Publishing Company, London. 1997.

[28] W. A. Brock, D. A. Hsieh and B. LeBaron, "Nonlinear Dynamics, Chaos and Instability: Statistical Theory and Economic Evidence,” MIT Press, London, 1991.

[29] P. L'Ecuyer, “Good Parameters and Implementations for Combined Multiple Recursive Random Number Generators," Operations Research, Vol. 47, No. 1, 1999, pp. 159-164. doi:10.1287/opre.47.1.159

[30] M. Matsumoto and T. Nishimura, "Mersenne Twister: A 623-Dimensionally Equidistributed Uniform Pseudo-Random Number Generator," ACM Transactions on Modeling and Computer Simulation, Vol. 8, No. 1, 1998, pp. 330. doi:10.1145/272991.272995

[31] C. I. Lee, K. C. Gleason and I. Mathur, "A Comprehensive Examination of the Compass Rose Pattern in Futures Markets," Journal of Futures Markets, Vol. 19, No. 5, 1999, pp. 541-564. doi:10.1002/(SICI)1096-9934(199908)19:5<541::AID-F

\section{$\mathrm{UT3}>3.0 . \mathrm{CO} ; 2-7$}

[32] E. Wang, R. Hudson and K. Keasey, "Tick Size and the Compass Rose: Further Insights,” Economics Letters, Vol. 68, No. 2, 2000, pp. 119-125. doi:10.1016/S0165-1765(00)00237-8

[33] D. Ruelle, "Deterministic Chaos: The Science and the Fiction," Proceedings of the Royal Society of London, Vol. 427, No. 1873, 1991, pp. 241-248.

[34] J. B. Ramsay and H. J. Yuan, "Bias and Error Bars in Dimension Calculations and Their Evaluation in Some Simple Models,” Physics Letters A, Vol. 134, No. 5, 1989, pp. 287-297. doi:10.1016/0375-9601(89)90638-5

[35] J. B. Ramsay and H. J. Yuan, “The Statistical Properties of Dimension Calculations Using Small Data Sets,” Nonlinearity, Vol. 3, No. 1, 1990, pp. 155-175. doi:10.1088/0951-7715/3/1/009

[36] K. C. Gleason, C. I. Lee and I. Mathur, “An Explanation for the Compass Rose Pattern,” Economics Letters, Vol. 68, No. 2, 2000, pp. 127-133. doi:10.1016/S0165-1765(00)00252-4

[37] M. D. McKenzie and A. Frino, “The Tick/Volatility Ratio as a Determinant of the Compass Rose: Empirical evidence from decimalisation on the NYSE," Accounting and Finance, Vol. 43, No. 3, 2003, pp. 331-344. doi:10.1111/j.1467-629x.2003.00094.x 